\title{
Integrated generation of complex optical quantum states and their coherent control
}

Piotr Roztocki, Michael Kues, Christian Reimer, Luis Romero Cortés, Stefania Sciara, et al.

Piotr Roztocki, Michael Kues, Christian Reimer, Luis Romero Cortés, Stefania Sciara, Benjamin Wetzel, Yanbing Zhang, Alfonso Cino, Sai T. Chu, Brent E. Little, David J. Moss, Lucia Caspani, José Azaña, Roberto Morandotti, "Integrated generation of complex optical quantum states and their coherent control," Proc. SPIE 10456, Nanophotonics Australasia 2017, 104561A (2 January 2018); doi: 10.1117/12.2286435 


\title{
Integrated generation of complex optical quantum states and their coherent control
}

\author{
Piotr Roztocki ${ }^{1}$, Michael Kues ${ }^{1,2}$, Christian Reimer ${ }^{1}$, Luis Romero Cortés ${ }^{1}$, Stefania Sciara ${ }^{1,3}$, \\ Benjamin Wetzel ${ }^{1,4}$, Yanbing Zhang, ${ }^{1}$, Alfonso Cino ${ }^{3}$, Sai T. Chu ${ }^{5}$, Brent E. Little ${ }^{6}$, David J. Moss ${ }^{7}$, \\ Lucia Caspani ${ }^{8,9}$, José Azaña ${ }^{1}$ \& Roberto Morandotti ${ }^{1,10,11}$ \\ ${ }^{1}$ INRS-EMT, 1650 Boulevard Lionel-Boulet, Varennes, Québec, J3X 1S2, Canada; ${ }^{2}$ School of \\ Engineering, University of Glasgow, Rankine Building, Oakfield Avenue, Glasgow G12 8LT, UK; \\ ${ }^{3}$ Department of Energy, Information Engineering and Mathematical Models, University of Palermo, \\ Palermo, Italy; ${ }^{4}$ School of Mathematical and Physical Sciences, University of Sussex, Falmer, \\ Brighton BN1 9RH, UK; ${ }^{5}$ Department of Physics and Material Science, City University of Hong \\ Kong, Tat Chee Avenue, Hong Kong, China; ${ }^{6}$ State Key Laboratory of Transient Optics and \\ Photonics, Xi' an Institute of Optics and Precision Mechanics, Chinese Academy of Science, Xi'an, \\ China; ${ }^{7}$ Centre for Micro Photonics, Swinburne University of Technology, Hawthorn, Victoria 3122, \\ Australia; ${ }^{8}$ Institute of Photonics, Department of Physics, University of Strathclyde, Glasgow G1 \\ 1RD, UK; ${ }^{9}$ Institute of Photonics and Quantum Sciences, Heriot-Watt University, Edinburgh EH14 \\ 4AS, UK; ${ }^{10}$ Institute of Fundamental and Frontier Sciences, University of Electronic Science and \\ Technology of China, Chengdu 610054, China; ${ }^{11}$ National Research University of Information \\ Technologies, Mechanics and Optics, St Petersburg, Russia.
}

\begin{abstract}
Complex optical quantum states based on entangled photons are essential for investigations of fundamental physics and are the heart of applications in quantum information science. Recently, integrated photonics has become a leading platform for the compact, cost-efficient, and stable generation and processing of optical quantum states. However, onchip sources are currently limited to basic two-dimensional (qubit) two-photon states, whereas scaling the state complexity requires access to states composed of several $(>2)$ photons and/or exhibiting high photon dimensionality. Here we show that the use of integrated frequency combs (on-chip light sources with a broad spectrum of evenly-spaced frequency modes) based on high-Q nonlinear microring resonators can provide solutions for such scalable complex quantum state sources. In particular, by using spontaneous four-wave mixing within the resonators, we demonstrate the generation of bi- and multi-photon entangled qubit states over a broad comb of channels spanning the S, C, and L telecommunications bands, and control these states coherently to perform quantum interference measurements and state tomography. Furthermore, we demonstrate the on-chip generation of entangled high-dimensional (quDit) states, where the photons are created in a coherent superposition of multiple pure frequency modes. Specifically, we confirm the realization of a quantum system with at least one hundred dimensions. Moreover, using off-the-shelf telecommunications components, we introduce a platform for the coherent manipulation and control of frequencyentangled quDit states. Our results suggest that microcavity-based entangled photon state generation and the coherent control of states using accessible telecommunications infrastructure introduce a powerful and scalable platform for quantum information science.
\end{abstract}

Keywords: Quantum optics; Integrated photonics; Frequency combs; Nonlinear processes, spontaneous four-wave mixing

\section{INTRODUCTION}

Entangled optical quantum states represent a key resource for fundamental quantum science and applications such as quantum communications ${ }^{1}$, powerful processing and simulations ${ }^{2}$, as well as metrology and sensing ${ }^{3}$. Indeed, exploiting the full potential of photons for quantum technologies demands access to the custom preparation and coherent

Nanophotonics Australasia 2017, edited by James W. M. Chon, Baohua Jia, Proc. of SPIE Vol. 10456, 104561A · @ $2018 \mathrm{SPIE} \cdot$ CCC code: 0277-786X/18/\$18 · doi: 10.1117/12.2286435 
manipulation of complex optical quantum states. Their complexity can be augmented by increasing the number $\mathrm{N}$ of photons in the state and/or the photon dimensionality $\mathrm{D}$, where the state Hilbert space size scales as $\mathrm{D}^{\wedge} \mathrm{N}$.

While the low-footprint and stability of integrated photonics has made it a highly-competitive platform for the generation and processing of optical quantum states, complex states remain largely inaccessible. Specifically, the generation of multi-partite entangled states ${ }^{4}$ is experimentally very challenging and had not to date been achieved on an integrated platform. Likewise, using current approaches, the on-chip preparation of high-dimensional optical entanglement requires highly-complex and specialized components. Specifically, high-dimensional path-entanglement requires $\mathrm{D}$ coherently-excited identical sources and complicated circuits of beam-splitters ${ }^{5}$, while time-entanglement needs elaborate multi-arm interferometers ${ }^{6}$.

The frequency-domain offers a unique framework for the generation and manipulation of complex states within a single spatial mode, as shown by its recent exploitation in quantum frequency combs (QFC). Using integrated optics and telecommunication infrastructures, QFCs have recently been used to realize and coherently control the first on-chip sources of both multi-photon states ${ }^{7}$ and entangled high-dimensional states ${ }^{8-10}$, and as such, provide a promising framework for future quantum information technologies.

\section{QUANTUM FREQUENCY COMBS}

Integrated frequency combs are on-chip light sources with a broad spectrum of evenly-spaced frequency modes, and are emitted from a nonlinear micro-cavity by optically-exciting one of its resonances ${ }^{11}$. When this micro-cavity is operated below the optical parametric oscillation threshold, two-photon states can be generated. In third-order nonlinear microcavities, spontaneous four-wave mixing (SFWM) annihilates two excitation field photons and generates a signal and an idler photon ${ }^{12}$ (Figure 1). Both the signal and idler are created in a superposition of the neighboring spectrally-distinct frequency comb mode states, and are spectrally correlated due to the energy conservation of the SFWM process.

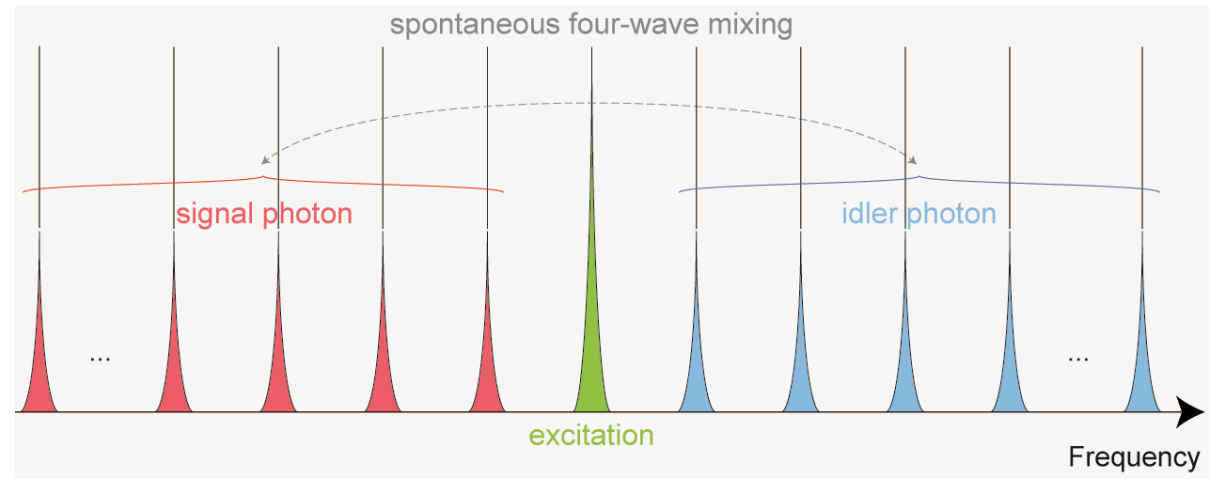

Figure 1. An excitation field is coupled to the integrated nonlinear micro-resonator and is made, through filtering, to only excite a single resonance. Spontaneous four-wave mixing mediates the annihilation of two excitation field photons and the generation of a daughter photon pair (signal and idler) superimposed over the neighboring frequency modes.

Thanks to cavity field enhancement, QFC sources deliver high quantum state generation rates at low excitation powers compared to plain-waveguide sources ${ }^{13}$. More importantly, this concept also provides a solution for state scalability and complexity by allowing several frequency modes (compatible with telecommunications wavelength-division multiplexing channels) to be accessible within a single waveguide spatial mode, and straightforward access to entanglement, superposition, and multi-partite states. Several multi-channel sources based on QFCs have already been demonstrated, among them combs of correlated photons ${ }^{12}$, cross-polarized photon pairs $^{14}$, entangled photon pairs ${ }^{7,15,16}$, multi-photon states ${ }^{7}$, and frequency-bin entangled $\operatorname{states}^{8-10}$.

\section{MULTI-PHOTON ENTANGLED STATE GENERATION AND CONTROL}

Through the double-pulse excitation of an integrated micro-ring cavity resonance, we demonstrate the generation of time-bin entangled photon pairs ${ }^{17}$ over the entire frequency comb spectrum (where the daughter photon pair is produced 
in a superposition of two discrete generation times, corresponding to the two coherent-but-separate pulsed excitations of the micro-ring). Stabilized fiber interferometers, comprised of standard fiber telecommunications elements, are used to both prepare the pulse pair and project the time-bin entangled qubits for processing. Using this scheme, we measure qubit entanglement with fidelities above $90 \%$ both on co- as well as cross-polarized photon pairs, compatible with future on-chip implementation of quantum information processing protocols. The measured density matrix of the two-photon state also agrees very well with the expected ideal state, confirmed by a measured fidelity of $96 \%$.

Finally, we note that in our quantum comb, the excitation field and the generated photons are intrinsically bandwidthmatched due to the resonance characteristics of the ring cavity, enabling the multiplication of Bell states and the generation of a four-photon time-bin entangled state. We confirm the generation of this four-photon entangled state through four-photon quantum interference with a measured visibility of $89 \%$ without background correction ${ }^{7}$. The measured density matrix of the four-photon entangled qubit state also reaches a fidelity of $64 \%$, comparable in quality to other, non-integrated four-photon states.

\section{HIGH-DIMENSIONAL ENTANGLED STATE GENERATION AND CONTROL}

We spectrally filtered a mode-locked laser to excite a single resonance of an integrated micro-ring resonator. The photon pairs generated cover multiple resonances due to the broad phase-matching condition, and are intrinsically generated as a high-dimensional superposition of resonance frequency mode states, and entangled due to the SFWM energy conservation condition. We then exploit a concatenation of standard telecommunications elements for the coherent manipulation of the state within a single spatial mode, exploiting such elements as programmable spectral phase filters and electro-optical phase modulators. The programmable filters are used to impose an arbitrary phase and amplitude mask on the frequency components of the quantum state, while phase modulation deterministically shifts and mixes the different frequency components in a designed manner.

The control scheme can then be used to implement arbitrary phase gates, as well as to perform deterministic highdimensional projection measurements. We first use these components to characterize the dimensionality of the state, and conclude, using joint spectral intensity ${ }^{18}$ and second-order coherence function ${ }^{19}$ measurements, that the quantum state has a Schmidt number of 10 and Hilbert space dimensionality of 100, which is formed by two entangled quDits with $\mathrm{D}=10$. We then validate our platform by measuring violations of a high-dimensional Bell inequality and performing quantum state tomography. Specifically, we record quantum interference for $\mathrm{D}=2,3$, and 4 , where we find visibilities of $83.7 \%, 86.6 \%$, and $86.4 \%$, respectively, all violating their respective high-dimensional Bell inequalities for $\mathrm{D}=2,3$, and 4 , respectively ${ }^{20}$. We then performed quantum state tomography to experimentally extract the state density matrices also for $\mathrm{D}=2,3$, and 4 , confirming that the experimental quantum states are very close to the ideal maximally entangled states with measured fidelities of $88.5 \%, 80.9 \%$, and $76.6 \%$ for $\mathrm{D}=2,3$, and 4 , respectively ${ }^{8}$.

\section{CONCLUSION}

Integrated quantum frequency combs based on third-order nonlinearities are shown to be a scalable and versatile platform for quantum state generation and for practical, optical quantum information processing. Our results indicate that microcavity-based multi-photon and high-dimensional entangled states and their time- and spectral-domain manipulation using standard telecommunications components can open up new venues for reaching the state complexities and processing capabilities required for meaningful quantum information science.

\section{ACKNOWLEDGEMENTS}

We thank R. Helsten for technical insights; P. Kung from QPS Photronics for the help and processing equipment; as well as QuantumOpus and N. Bertone of OptoElectronics Components for their support and for providing us with state-ofthe-art photon detection equipment. This work was made possible by the following funding sources: Natural Sciences and Engineering Research Council of Canada (NSERC) (Steacie, Strategic, Discovery, and Acceleration Grants Schemes, Vanier Canada Graduate Scholarships, USRA Scholarship); Mitacs (IT06530) and PBEEE (207748); MESI 
PSR-SIIRI Initiative; Canada Research Chair Program; Australian Research Council Discovery Projects (DP150104327); European Union's Horizon 2020 research and innovation program under the Marie Sklodowska-Curie grant (656607); CityU SRG-Fd program (7004189); Strategic Priority Research Program of the Chinese Academy of Sciences (XDB24030300); People Programme (Marie Curie Actions) of the European Union's FP7 Programme under REA grant agreement INCIPIT (PIOF-GA-2013-625466); Government of the Russian Federation through the ITMO Fellowship and Professorship Program (Grant 074-U 01); 1000 Talents Sichuan Program (China)

\section{REFERENCES}

[1] Kimble, H. J., “The quantum internet.," Nature 453(7198), 1023-1030 (2008).

[2] Knill, E., Laflamme, R. and Milburn, G. J., "A scheme for efficient quantum computation with linear optics," Nature 409(6816), 46-52 (2001).

[3] Israel, Y., Rosen, S. and Silberberg, Y., "Supersensitive Polarization Microscopy Using NOON States of Light," Phys. Rev. Lett. 112(10), 103604 (2014).

[4] Briegel, H. J. and Raussendorf, R., "Persistent Entanglement in Arrays of Interacting Particles," Phys. Rev. Lett. 86(5), 910-913 (2001).

[5] Schaeff, C., Polster, R., Lapkiewicz, R., Fickler, R., Ramelow, S. and Zeilinger, A., "Scalable fiber integrated source for higher-dimensional path-entangled photonic quNits," Opt. Express 20(15), 16145 (2012).

[6] Thew, R., Acin, A., Zbinden, H., Gisin, N., "Experimental realization of entangled qutrits for quantum communication," Quantum Inf. Comput. 4(2), 93 (2004).

[7] Reimer, C., Kues, M., Roztocki, P., Wetzel, B., Grazioso, F., Little, B. E., Chu, S. T., Johnston, T., Bromberg, Y., Caspani, L., Moss, D. J. and Morandotti, R., "Generation of multiphoton entangled quantum states by means of integrated frequency combs," Science (80-. ). 351(6278), 1176-1180 (2016).

[8] Kues, M., Reimer, C., Roztocki, P., Cortés, L. R., Sciara, S., Wetzel, B., Zhang, Y., Cino, A., Chu, S. T., Little, B. E., Moss, D. J., Caspani, L., Azaña, J. and Morandotti, R., "On-chip generation of high-dimensional entangled quantum states and their coherent control," Nature 546(7660), 622-626 (2017).

[9] Imany, P., Jaramillo-Villegas, J. A., Odele, O. D., Han, K., Qi, M., Leaird, D. E. and Weiner, A., "Demonstration of frequency-bin entanglement in an integrated optical microresonator," Conf. Lasers ElectroOptics 62(19), JTh5B.3, OSA, Washington, D.C. (2017).

[10] Roztocki, P., Kues, M., Reimer, C., Wetzel, B., Sciara, S., Zhang, Y., Cino, A., Little, B. E., Chu, S. T., Moss, D. J. and Morandotti, R., "Practical system for the generation of pulsed quantum frequency combs," Opt. Express 25(16), 18940 (2017).

[11] Del'Haye, P., Schliesser, A., Arcizet, O., Wilken, T., Holzwarth, R. and Kippenberg, T. J., “Optical frequency comb generation from a monolithic microresonator," Nature 450(7173), 1214-1217 (2007).

[12] Reimer, C., Caspani, L., Clerici, M., Ferrera, M., Kues, M., Peccianti, M., Pasquazi, A., Razzari, L., Little, B. E., Chu, S. T., Moss, D. J. and Morandotti, R., "Integrated frequency comb source of heralded single photons," Opt. Express 22(6), 6535-6546 (2014).

[13] Bonneau, D., Silverstone, J. W. and Thompson, M. G., "Silicon Quantum Photonics," [Silicon Photonics III], L. Pavesi and D. J. Lockwood, Eds., Springer Berlin Heidelberg, Berlin, Heidelberg, 41-82 (2016).

[14] Reimer, C., Kues, M., Caspani, L., Wetzel, B., Roztocki, P., Clerici, M., Jestin, Y., Ferrera, M., Peccianti, M., Pasquazi, A., Little, B. E., Chu, S. T., Moss, D. J. and Morandotti, R., "Cross-polarized photon-pair generation and bi-chromatically pumped optical parametric oscillation on a chip," Nat. Commun. 6, 8236 (2015).

[15] Grassani, D., Azzini, S., Liscidini, M., Galli, M., Strain, M. J., Sorel, M., Sipe, J. E. and Bajoni, D., "Micrometer-scale integrated silicon source of time-energy entangled photons," Optica 2(2), 88 (2015).

[16] Mazeas, F., Traetta, M., Bentivegna, M., Kaiser, F., Aktas, D., Zhang, W., Ramos, C. A., Ngah, L. A., Lunghi, T., Picholle, É., Belabas-Plougonven, N., Le Roux, X., Cassan, É., Marris-Morini, D., Vivien, L., Sauder, G., Labonté, L. and Tanzilli, S., "High-quality photonic entanglement for wavelength-multiplexed quantum communication based on a silicon chip," Opt. Express 24(25), 28731 (2016).

[17] Brendel, J., Gisin, N., Tittel, W. and Zbinden, H., "Pulsed Energy-Time Entangled Twin-Photon Source for Quantum Communication," Phys. Rev. Lett. 82(12), 2594-2597 (1999).

[18] Law, C. K., Walmsley, I. A. and Eberly, J. H., "Continuous Frequency Entanglement: Effective Finite Hilbert Space and Entropy Control,” Phys. Rev. Lett. 84(23), 5304-5307 (2000).

[19] Christ, A., Laiho, K., Eckstein, A., Cassemiro, K. N. and Silberhorn, C., "Probing multimode squeezing with 
correlation functions," New J. Phys. 13(3), 33027 (2011).

[20] Collins, D., Gisin, N., Linden, N., Massar, S. and Popescu, S., "Bell Inequalities for Arbitrarily HighDimensional Systems," Phys. Rev. Lett. 88(4), 40404 (2002). 